\title{
Estimation of genetic parameters for partial egg production periods by means of random regression models
}

\author{
G.C. Venturini ${ }^{1}$, D.A. Grossi ${ }^{1}$, S.B. Ramos ${ }^{1}$, V.A.R. Cruz ${ }^{1}$, C.G. Souza ${ }^{1}$, \\ M.C. Ledur ${ }^{2}$, L. EI Faro ${ }^{3}$, G.S. Schmidt ${ }^{2}$ and D.P. Munari ${ }^{1}$ \\ ${ }^{1}$ Departamento de Ciências Exatas, Faculdade de Ciências Agrárias e \\ Veterinárias, Universidade Estadual Paulista "Júlio de Mesquita Filho", \\ Jaboticabal, SP, Brazil \\ ${ }^{2}$ Empresa Brasileira de Pesquisa Agropecuária - Embrapa Suínos e Aves, \\ Concórdia, SC, Brazil \\ ${ }^{3}$ Agência Paulista de Tecnologia dos Agronegócios, \\ Pólo Regional Centro Leste, Ribeirão Preto, SP, Brazil \\ Corresponding author: D.P. Munari \\ E-mail:danisio@fcav.unesp.br
}

Genet. Mol. Res. 11 (3): 1819-1829 (2012)

Received September 12, 2011

Accepted March 6, 2012

Published July 10, 2012

DOI http://dx.doi.org/10.4238/2012.July.10.18

\begin{abstract}
We estimated genetic parameters for egg production in different periods by means of random regression models, aiming at selection based on partial egg production from a generation of layers. The production was evaluated for each individual by recording the number of eggs produced from 20 to 70 weeks of age, with partial records taken every three weeks for a total of 17 periods. The covariance functions were estimated with a random regression model by the restricted maximum likelihood method. A model composed of third-order polynomials for the additive effect, ninth-order polynomials for the permanent environment, and a residual variance structure with five distinct classes, was found to be most suitable for adjusting the egg production data for laying hens. The heritability estimates varied from 0.04 to 0.14 . The genetic correlations were all positive, varying from 0.10 to 0.99 . Selection applied in partial egg production periods will result in greater genetic profit for the adjacent
\end{abstract}


periods. However, as the distance in time between periods increases, selection becomes less efficient. Selection based on the second period ( 23 to 25 weeks of age), where greater heritability was estimated, would note benefit the final egg-laying cycle periods.

Key words: Longitudinal data; Poultry; Random regression

\section{INTRODUCTION}

Partial egg production records can be considered as longitudinal data and can be analyzed during the life of an animal by using linear (Al-Samarai et al., 2008) or nonlinear functions (Fialho and Ledur, 1997; Grossman et al., 2000) incorporated into repeatability (Wolc et al., 2007) or multitrait (Pires et al., 2002; Wolc et al., 2008) models. Random regression models (RRMs) are linear models, also known as finite-dimension models, are used to evaluate initial milk production in dairy cows (Jamrozik et al., 1997). They can also be used to evaluate egg production in laying hens when partial laying periods are considered.

For animal evaluations, RRMs consider the average production curve of the studied population, the individual random curves obtained because of the deviation from the average curve, and the relationship between the animals. Because they use partial periods and individual production curves, these models can evaluate egg production in animals that show changes in production over their lifetime. Traits that can be measured repeatedly during the lifetime of an animal, such as growth rate or egg or milk production rate, show genetic correlations between measurements that often decrease as the time between measurements increases.

In laying hens, egg production begins on average at the age of 20 weeks (Szydlowski and Szwaczkowski, 2001). The age, in days, when a bird begins the productive cycle defines the sexual maturity of the hen. Peak egg production is reached at approximately 26 weeks of age (Fialho and Ledur, 1997). Subsequently, the production gradually decreases until approximately 70 weeks of age as a function of the bird's physiological condition, age, and genetic constitution, which has been linked to the occurrence of natural changes, egg hatching, or egg production reduction. Factors such as age at sexual maturity and laying persistence directly contribute to the rate of egg production in the first cycle.

The use of RRMs for the genetic evaluation of poultry has not yet been reported, and studies focusing especially on the optimization of the definition of the length of the egg-laying period and choice order of polynomials can be performed using these models. Hence, the objective of this study was to apply an RRM to estimate the genetic parameters for the partial production of eggs in a White Leghorn strain.

\section{MATERIAL AND METHODS}

The egg production records of females from the seventh generation of a White Leghorn strain called "CC," were used. These records were maintained by the Poultry Genetic Improvement Program of Embrapa Suínos e Aves, Concórdia, Santa Catarina, Brazil. This generation was produced by breeding 43 roosters with 232 hens ( 5 hens per rooster, on average) in a hierarchical scheme by using artificial insemination. The eggs were incubated for intervals of 15 days ( 3 hatches), and the birds were identified by banding. $\mathrm{CC}$ is a pure line 
mainly selected for egg and egg weight, feed conversion, hatchability, sexual maturity, fertility, viability, egg quality, and reduced body weight (Savegnago et al., 2011).

The egg production was evaluated individually by monitoring the number of eggs obtained from 20 to 70 weeks of age for each hen. In all, 17 partial periods (P1 to P17) were defined, with each period consisting of 3 weeks. The eggs were collected over 5 days of the week. The average production per period of 3 weeks varied from 10.12 (P1) to 13.41 (P4) eggs per 15 days (Table 1). For data analysis, the following were excluded from the archives: females obtained from dams with less than 2 progenies, females with production numbers of less than 63 eggs (which represents $30 \%$ of the laying rate, considering the average total production of the population), and females that died during the total productive period. The final number of females studied was 1494.

The genetic parameters were estimated using covariance functions and an RRM by applying the restricted maximum likelihood method (option DXMRR of the DFREML statistical program) (Meyer, 1998a). The initial values requested by the program were obtained from the literature (Anang et al., 2000).

The statistical model included the fixed effects of incubation and average production trajectory, random genetic additive effects $(k a)$, effects of the permanent direct environment $(k c)$, and residues $(e)$. The fixed trajectory was adjusted by a cubic Legendre polynomial. The random trajectories were modeled using the Legendre orthogonal polynomials, and the order of the regression coefficients was varied. First, the residual variance was modeled by classes (Meyer, 1998b), fixing the order of $k a$ and $k c$ to 3. Four models were analyzed: one with residual variance homogeneity $(e=1)$ and 3 with heterogeneity $(e=4, e=5$, and $e=17)$. The $e=4$ and $e=5$ structures were grouped on the basis of residual variance similarities of each period for the $e=17$ model. Thus, for $e=4$, the following periods were considered: the first, second to fifth, sixth to eleventh, and twelfth to seventeenth residues. For $e=5$, the first, second to fifth, sixth, seventh to eleventh, and twelfth to seventeenth residues were considered. After selecting the best residual variance structure, 12 different models were tested by varying the $k a$ order from 3 to 4 and the $k c$ order from 3 to 9 coefficients. The evaluated models are described in Table 2.

The general random regression model can be written as follows:

$$
y_{i j}=\mathrm{I} n c+\sum_{m=0}^{k b-1} \beta_{m} \phi_{m}\left(t_{i}\right)+\sum_{m=0}^{k a-1} \alpha_{j m} \phi_{m}\left(t_{i j}\right)+\sum_{m=0}^{k c-1} \delta_{j m} \phi_{m}\left(t_{i j}\right)+E_{i j},(\text { Equation } 1)
$$

where $y_{i j}$ is the egg production in the $i$-th period belonging to the $j$-th animal; Inc is the fixed incubation effect; $\beta_{m}$ is the regression coefficient modeling the average population trajectory; $\phi_{m}\left(t_{i}\right)$ is the regression function of the $k b$-th order that describes the average population curve according to the production period $\left(t_{i}\right) ; \phi_{m}\left(t_{j}\right)$ is the regression function that describes the trajectories of each individual $j$ in accordance with the period $\left(t_{i}\right)$ for the random additive genetic effect and the direct permanent environment; $\alpha_{j n}$ and $\delta_{j m}$ are the random genetic additive repressors and the direct permanent environment for each animal; $k b, k a$, and $k c$ are the polynomial orders used for the effects described above; and $E_{i j}$ is the random error associated with each age $i$ of animal $j$. The general animal model can be represented by the following equation: 


\section{$y=X b+Z a+W c+e$,}

(Equation 2)

where $y$ is the phenotypic observation vector; $b$ is the fixed effect of incubation and the average trajectory regression coefficient vector; $a$ is the vector of random genetic additive effects; $c$ is the vector for random coefficients of the direct permanent environment; $e$ is the residue vector; and $X, \mathrm{Z}$, and $W$ are the incidence matrices corresponding to the fixed effect, random genetic additive, and direct permanent environment, respectively. The presuppositions in relation to the model are as follows:

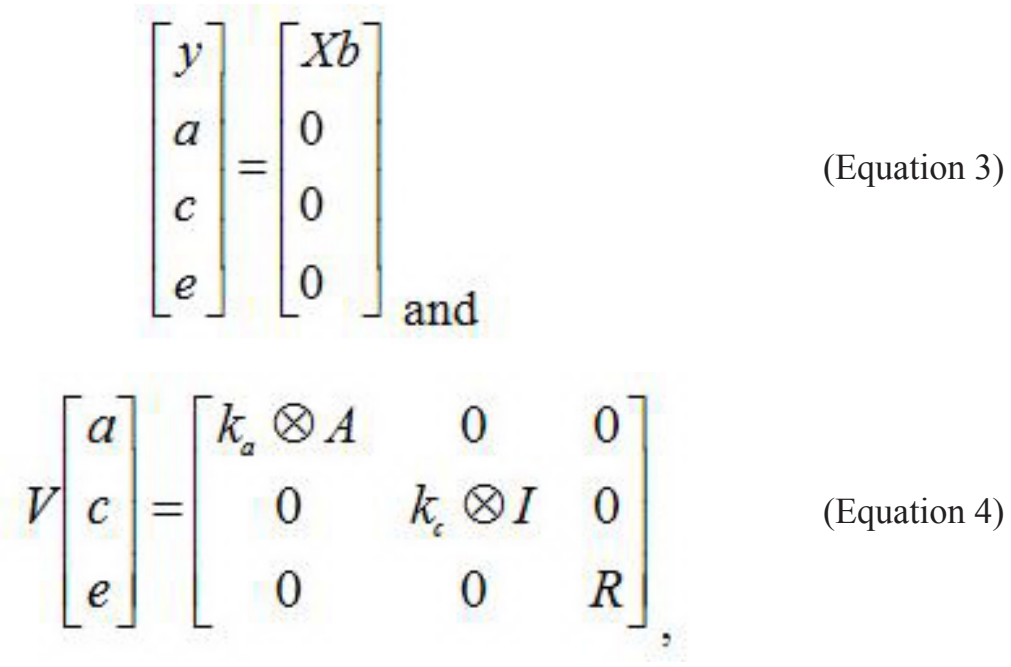

where $k_{a}$ and $k_{c}$ are the variance and covariance matrices between the random regression coefficients for the direct genetic additive and direct permanent environment effects; $A$ is the relationship matrix among the individuals $(12,006$ animals $) ; I$ is the identity matrix; $\otimes$ is the Kroenecker product between matrices; and $R$ represents a diagonal block matrix containing the residual variances. In this model, no correlation between the random regression coefficients was assumed for the direct genetic additive and direct permanent environment effects.

The adjusted quality of these models was compared using the Akaike information criteria (AIC), the Schwarz Bayesian information criteria (BIC) (Nuñes-Antón and Zimmerman, 2000), the logarithmic probability function values $(\log L)$, and the likelihood ratio test (LRT) for nested models. Two models were considered nested when 2 of the 3 polynomial orders were fixed (genetic and permanent or residual environment effects) in order to complete the LRT. Thus, the LRT allowed the verification of whether the difference in the parameter quantity was significant by using the chi-square statistical test. The number of degrees of freedom for this test was obtained using the difference between the numbers of model parameters involved in the test. When the differences between the models were not significant $(\mathrm{P}>0.05)$, the less parameterized model was considered. The models were compared and the model with a better adjusted quality was determined by obtaining the value closest to zero by using $\log L$. 
Subsequently, the lowest values of AIC and BIC were considered. However, among the models indicated by the tests, the distribution of the genetic correlation estimates among partial production periods was determined in order to identify the best model.

\section{RESULTS AND DISCUSSION}

The production averages observed according to laying period (P1 to $\mathrm{P} 17)$ are presented in Table 1. The periods P1, P16, and P17 (from 20 to 22, 65 to 67, and 68 to 70 weeks of age, respectively), representing the initial and final production periods, had the lowest production averages, with $10.12 \pm 4.12,10.66 \pm 3.87$, and $10.15 \pm 3.94$ eggs produced and greater variation coefficients (VCs) of $40.71,36.30$, and $38.82 \%$, respectively (Table 1 ). Anang et al. (2002) observed similar variations for the initial and final production periods. In the initial period, the phenotypic variation can be attributed to the differences in the age of sexual maturity, whereas the laying persistence variation, hatching occurrence, and natural change might influence the phenotypic values of the final periods.

Table 1. Observed averages, the respective standard deviation (SD) and variation coefficient (VC) for egg production by partial period (P1 to P17) obtained every three weeks of age.

\begin{tabular}{|c|c|c|c|c|}
\hline Period (P) & Week of age & Average & SD & $\mathrm{VC}(\%)$ \\
\hline 1 & 20 to 22 & 10.12 & 4.12 & 40.71 \\
\hline 2 & 23 to 25 & 13.10 & 2.49 & 19.02 \\
\hline 3 & 26 to 28 & 12.99 & 2.46 & 18.98 \\
\hline 4 & 29 to 31 & 13.41 & 2.43 & 18.14 \\
\hline 5 & 32 to 34 & 13.30 & 2.72 & 20.49 \\
\hline 6 & 35 to 37 & 13.11 & 2.98 & 22.70 \\
\hline 7 & 38 to 40 & 13.25 & 2.87 & 21.65 \\
\hline 8 & 41 to 43 & 13.04 & 2.80 & 21.50 \\
\hline 9 & 44 to 46 & 12.85 & 2.96 & 23.01 \\
\hline 10 & 47 to 49 & 12.62 & 3.11 & 24.67 \\
\hline 11 & 50 to 52 & 12.38 & 3.22 & 26.02 \\
\hline 12 & 53 to 55 & 12.16 & 3.28 & 27.01 \\
\hline 13 & 56 to 58 & 11.79 & 3.45 & 29.28 \\
\hline 14 & 59 to 61 & 11.58 & 3.60 & 31.05 \\
\hline 15 & 62 to 64 & 11.12 & 3.81 & 34.26 \\
\hline 16 & 65 to 67 & 10.66 & 3.87 & 36.30 \\
\hline 17 & 68 to 70 & 10.15 & 3.94 & 38.82 \\
\hline
\end{tabular}

The production peak occurred during P4 (from 29 to 31 weeks of age), since this was the period with the greatest observed egg production average $(13.41 \pm 2.43)$. This average was greater than the average reported by Fialho and Ledur (1997) at 26 weeks of age. A result similar to that obtained in this study was found by Luo et al. (2007) for a line of female broilers; they observed that the egg production peak was reached around 28 to 29 weeks of age. The egg production started to decrease from P9 (from 44 to 46 weeks of age; average, $12.85 \pm 2.96$ eggs). Anang et al. (2002) observed that this decrease in the number of eggs began around 36 weeks of age. The lines for which the production reduction occurred at younger ages showed weaker laying persistence.

The results of the tests performed to evaluate the adjusted quality of the studied models are described in Table 2. In the evaluation of the best residual variance structure (models $1,2,3$, and 4), the BIC indicated model $3(k a=3, k c=3$, and $e=5)$ and the $\log L$ and AIC indicated model $4(k a=3, k c=3$, and $e=17)$. According to the LRT, models 3 and 4 differed 
significantly $(\mathrm{P}<0.01)$ with regard to the quantity of parameters. Thus, the residual variance structures were fixed in 5 classes (the first, second to fifth, sixth, seventh to eleventh, and twelfth to seventeenth residue) because the BIC value indicated that the less parameterized model $(e=5)$ should be used.

Table 2. Polynomial order for additive genetic effects $(k a)$, permanent environment order $(k c)$ and residues $(e)$ with the variance homogeneity and variance classes, the number of parameters (p), the probability function logarithms $(\log L)$, the Akaike information criterion (AIC), the Schwarz Bayesian information criterion (BIC) and the likelihood ratio test (LRT) for nested models.

\begin{tabular}{|c|c|c|c|c|c|c|c|c|}
\hline \multirow[t]{2}{*}{ Model } & \multicolumn{3}{|c|}{ Polynomial order } & \multirow[t]{2}{*}{$\mathrm{p}$} & \multirow[t]{2}{*}{$\log L$} & \multirow[t]{2}{*}{$\mathrm{AIC}$} & \multirow[t]{2}{*}{$\mathrm{BIC}$} & \multirow[t]{2}{*}{ LRT* } \\
\hline & $k a$ & $k c$ & $e$ & & & & & \\
\hline 1 & 3 & 3 & 1 & 13 & -38405.68 & 76837.36 & 76943.27 & \\
\hline 2 & 3 & 3 & 4 & 16 & -33188.83 & 66409.65 & 66539.54 & $19.63 * * *$ \\
\hline 3 & 3 & 3 & 5 & 17 & -33169.20 & 66372.40 & 66510.41 & $26.93 * *$ \\
\hline 4 & 3 & 3 & 17 & 29 & -33142.27 & 66342.55 & 66577.97 & \\
\hline 5 & 3 & 4 & 5 & 21 & -32982.86 & 66007.72 & 66178.20 & $80.81 * * *$ \\
\hline 6 & 3 & 5 & 5 & 26 & -32902.05 & 65856.10 & 66067.17 & $89.59^{* * *}$ \\
\hline 7 & 3 & 6 & 5 & 32 & -32812.46 & 65688.91 & 65948.69 & $44.94 * * *$ \\
\hline 8 & 3 & 7 & 5 & 39 & -32767.52 & 65613.04 & 65929.65 & $59.10 * * *$ \\
\hline 9 & 3 & 8 & 5 & 47 & -32708.42 & 65510.85 & 65892.40 & $20.33^{*}$ \\
\hline 10 & 3 & 9 & 5 & 56 & -32688.09 & 65488.19 & 65942.80 & \\
\hline 11 & 4 & 4 & 5 & 25 & -32964.23 & 65978.47 & 66181.42 & $88.73^{* * *}$ \\
\hline 12 & 4 & 5 & 5 & 30 & -32875.50 & 65811.00 & 66054.54 & $108.77 * * *$ \\
\hline 13 & 4 & 6 & 5 & 36 & -32766.73 & 65605.46 & 65897.71 & $41.23 * * *$ \\
\hline 14 & 4 & 7 & 5 & 43 & -32725.50 & 65537.00 & 65886.08 & $56.10 * * *$ \\
\hline 15 & 4 & 8 & 5 & 51 & -32669.40 & 65440.79 & 65854.82 & $18.21^{*}$ \\
\hline 16 & 4 & 9 & 5 & 60 & -32651.19 & 65422.38 & 65909.46 & \\
\hline
\end{tabular}

Values in bold indicate the best model based on AIC and BIC. * $(\mathrm{P}<0.05), * *(\mathrm{P}<0.01)$ and $* * *(\mathrm{P}<0.001)$ by the $\chi^{2}$ test.

For studying the optimum adjustment model, the orders of $e=5, k a=3$ (models 5 to 10 ) and $k a=4$ (models 11 to 16 ) were fixed by varying the $k c$ order for each model from 4 to 9 . When these models were evaluated using LRT, significant differences $(\mathrm{P}<0.05)$ were found between all the nested models. Comparisons between 10 and 16 models (4 degrees of freedom; $\left.\chi^{2}=36.9\right)$ and between 9 and 15 ( 4 degrees of freedom; $\left.\chi^{2}=39\right)$ were also significant $(\mathrm{P}<0.001)$ according to the LRT. Therefore, the LRT indicated that the most parameterized model would have the best quality adjustment. From the result of LRT and $\log \mathrm{L}$, the optimum adjustment model was found to be parameterized (model 16, with $k a=4, k c=9$, and $e=5$ ).

According to the AIC, the model most suitable for adjusting the egg production from among those with 5 residual variances classes (Table 2, model 16) was the 60-parameter model, with order 4 for $k a$ and 9 for $k c(k a=4, k c=9$, and $e=5)$. The BIC test, which penalizes more parameterized models, indicated model 15 containing 4 and 8 orders for $k a$ and $k c$, respectively $(k a=4, k c=8$, and $e=5)$, with the same residual variance structure.

The estimated genetic correlations for the models indicated by the AIC and BIC showed greater oscillations for the models with the fourth-order polynomials for an equal genetic additive effect, suggesting that it would be preferable to use a model with fewer parameters $(k a=3)$. According to some authors (Meyer, 1998b; El Faro et al., 2008), the surfaces described for genetic correlations must be soft and should not show large oscillations. The same applies to heritability estimates. 
The more parameterized models $(k a=4)$ in this study presented surfaces with greater oscillations for the genetic correlation estimates over time (Figure 1). This showed that production in hens similar in age was less genetically correlated than in those distant in age. This type of standard, according to Meyer (1998b), indicates that the model would be superparameterized. Thus, the best order for the additive genetic effect was 3 and not 4, as indicated by the AIC and BIC.
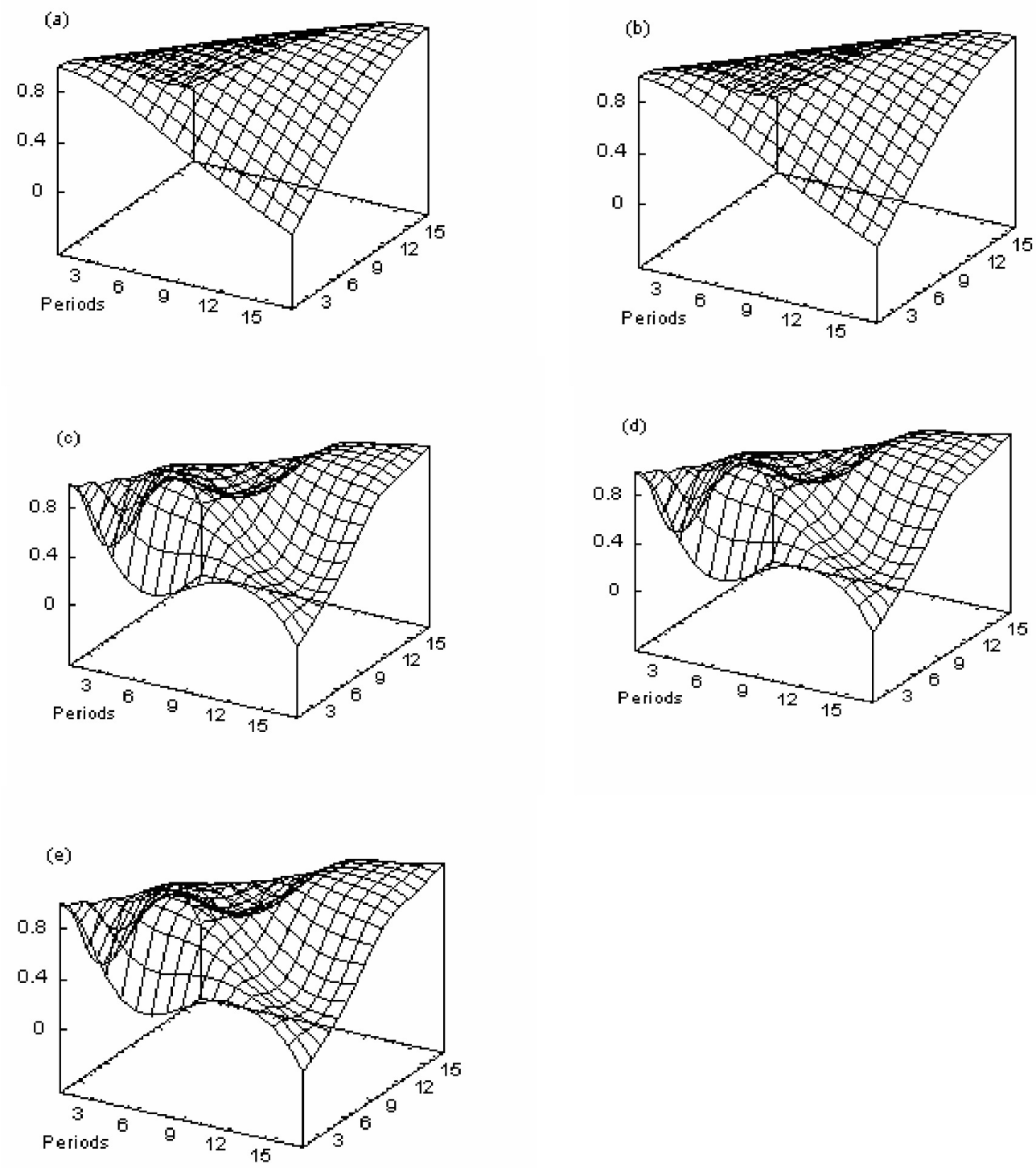

Figure 1. Genetic correlation estimates between the partial periods of egg production for the randon regression models (a) $\mathrm{ka} 3 \mathrm{kc} 8 \mathrm{e} 5$, (b) $\mathrm{ka} 3 \mathrm{kc} 9 \mathrm{e} 5$, (c) $\mathrm{ka} 4 \mathrm{kc} 7 \mathrm{e} 5$, (d) $\mathrm{ka} 4 \mathrm{kc} 8 \mathrm{e} 5$, and (e) $\mathrm{ka} 4 \mathrm{kc} 9 \mathrm{e} 5$, where $k a, k c$ and $e$ are the polynomial order for genetic effects, the permanent environment and the residue classes, respectively. 
Therefore, the model containing 56 parameters was indicated, that is, the model for evaluating egg production in laying hens having a lower-order polynomial for the genetic additive variance $(k a=3)$ and ninth-order polynomial for the permanent environment variance $(k c=9)$ was selected. This model presented genetic correlation estimates with a straightforward biological explanation, despite possessing a softer surface compared with other models (Figure 1).

Estimates of phenotypic, genetic additive, and permanent environment variances for the indicated model $\left(\mathrm{k}_{\mathrm{a}=3}, \mathrm{k}_{\mathrm{c}=9}\right.$, and $\left.\mathrm{k}_{\mathrm{e}=5}\right)$ are described in Figure 2. For the phenotypic variances, differences were noted between the studied periods, mainly for the first period ( $\mathrm{P} 1$, from 20 to 22 weeks of age) in relation to the others. This difference might have occurred because of the great influence of age on the sexual maturity and egg production in this period. Differences among phenotypic variances between lactation periods have also been observed by El Faro et al. (2008) and Bignardi et al. (2008) in dairy cows. For the genetic additive variances and permanent environment, no variations in the estimates were observed between the studied egg production periods. Bignardi et al. (2008) also did not find large genetic additive and permanent environment variations between the analyzed milk production periods.
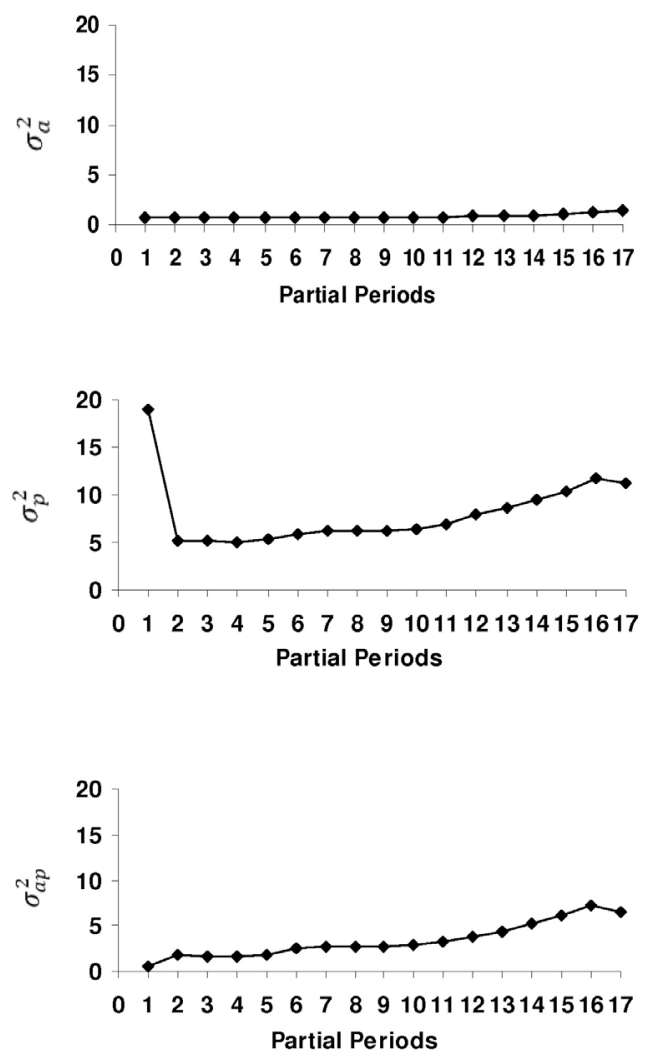

Figure 2. Line diagrams for the estimates of genetic additive $\left(\sigma_{a}^{2}\right)$, phenotypic $\left(\sigma_{p}^{2}\right)$ and permanent environment $\left(\sigma_{a p}^{2}\right)$ variance obtained for the random regression model ka3kc9e5 (lozenges), where $k a, k c$ and $e$ are the polynomial order for genetic effects, the permanent environment and the residue classes, respectively. 
The heritability estimates for the indicated model $\left(\mathrm{k}_{\mathrm{a}=3}, \mathrm{k}_{\mathrm{c}=9}\right.$, and $\left.\mathrm{k}_{\mathrm{e}=5}\right)$ varied from 0.04 in P1 to 0.14 in P2 (Table 3). The heritability estimates for the other periods showed little variation. Low heritability values $(0.00 \pm 0.00$ to $0.16 \pm 0.01)$ for the initial egg production period were also reported by Anang et al. (2001). Luo et al. (2007) showed that egg production heritability estimates varied from 0.16 (from 26 to 27 weeks of age) to 0.54 (62 weeks of age). However, when the same authors grouped the studied periods (from 26 to 65 weeks of age) in months, the heritability estimates varied from 0.03 (tenth month of production) to 0.21 (second production month), which were similar to those obtained in the present study.

Table 3. Estimates of the genetic correlation ( $r_{a}$, above the diagonal), the phenotypic value $(r$, below the diagonal) and the heritability (diagonal, in bold) of the chosen model (ka3kc9e5) among the partial periods of egg production $(\mathrm{P})$.

\begin{tabular}{rccccccccccccccccc}
\hline $\mathrm{P}$ & 1 & 2 & 3 & 4 & 5 & 6 & 7 & 8 & 9 & 10 & 11 & 12 & 13 & 14 & 15 & 16 & 17 \\
\hline 1 & $\mathbf{0 . 0 4}$ & 0.99 & 0.97 & 0.93 & 0.87 & 0.81 & 0.74 & 0.66 & 0.60 & 0.53 & 0.46 & 0.39 & 0.32 & 0.26 & 0.20 & 0.15 & 0.10 \\
2 & 0.11 & $\mathbf{0 . 1 4}$ & 0.99 & 0.97 & 0.93 & 0.88 & 0.82 & 0.76 & 0.69 & 0.62 & 0.56 & 0.49 & 0.42 & 0.36 & 0.29 & 0.23 & 0.18 \\
3 & 0.07 & 0.41 & $\mathbf{0 . 1 3}$ & 0.99 & 0.97 & 0.93 & 0.89 & 0.84 & 0.78 & 0.72 & 0.65 & 0.58 & 0.52 & 0.45 & 0.38 & 0.32 & 0.26 \\
4 & 0.05 & 0.32 & 0.41 & $\mathbf{0 . 1 3}$ & 0.99 & 0.97 & 0.94 & 0.89 & 0.847 & 0.79 & 0.73 & 0.67 & 0.61 & 0.54 & 0.47 & 0.41 & 0.34 \\
5 & 0.04 & 0.27 & 0.32 & 0.41 & $\mathbf{0 . 1 2}$ & 0.99 & 0.97 & 0.94 & 0.90 & 0.86 & 0.81 & 0.75 & 0.69 & 0.62 & 0.56 & 0.49 & 0.42 \\
6 & 0.03 & 0.24 & 0.25 & 0.34 & 0.47 & $\mathbf{0 . 1 0}$ & 0.99 & 0.98 & 0.95 & 0.91 & 0.87 & 0.82 & 0.76 & 0.70 & 0.64 & 0.57 & 0.50 \\
7 & 0.03 & 0.23 & 0.21 & 0.29 & 0.42 & 0.52 & $\mathbf{0 . 1 0}$ & 0.99 & 0.98 & 0.95 & 0.92 & 0.88 & 0.83 & 0.77 & 0.71 & 0.65 & 0.58 \\
8 & 0.03 & 0.20 & 0.20 & 0.25 & 0.35 & 0.45 & 0.52 & $\mathbf{0 . 1 0}$ & 0.99 & 0.98 & 0.95 & 0.92 & 0.88 & 0.83 & 0.78 & 0.72 & 0.65 \\
9 & 0.04 & 0.17 & 0.19 & 0.22 & 0.28 & 0.34 & 0.43 & 0.51 & $\mathbf{0 . 1 1}$ & 0.99 & 0.98 & 0.96 & 0.92 & 0.88 & 0.83 & 0.78 & 0.72 \\
10 & 0.05 & 0.13 & 0.18 & 0.20 & 0.22 & 0.26 & 0.34 & 0.44 & 0.52 & $\mathbf{0 . 1 1}$ & 0.99 & 0.98 & 0.96 & 0.92 & 0.88 & 0.84 & 0.78 \\
11 & 0.06 & 0.11 & 0.15 & 0.18 & 0.20 & 0.22 & 0.26 & 0.35 & 0.46 & 0.55 & $\mathbf{0 . 1 1}$ & 0.99 & 0.98 & 0.96 & 0.92 & 0.88 & 0.84 \\
12 & 0.05 & 0.11 & 0.12 & 0.16 & 0.18 & 0.20 & 0.23 & 0.28 & 0.37 & 0.48 & 0.56 & $\mathbf{0 . 1 0}$ & 0.99 & 0.98 & 0.96 & 0.92 & 0.89 \\
13 & 0.04 & 0.12 & 0.11 & 0.14 & 0.17 & 0.20 & 0.23 & 0.26 & 0.33 & 0.41 & 0.50 & 0.57 & $\mathbf{0 . 1 0}$ & 0.99 & 0.98 & 0.96 & 0.93 \\
14 & 0.02 & 0.12 & 0.12 & 0.12 & 0.15 & 0.19 & 0.23 & 0.26 & 0.31 & 0.36 & 0.43 & 0.50 & 0.59 & $\mathbf{0 . 1 0}$ & 0.99 & 0.98 & 0.96 \\
15 & 0.02 & 0.11 & 0.12 & 0.12 & 0.14 & 0.17 & 0.22 & 0.26 & 0.30 & 0.33 & 0.38 & 0.44 & 0.54 & 0.63 & $\mathbf{0 . 1 0}$ & 0.99 & 0.98 \\
16 & 0.03 & 0.10 & 0.11 & 0.13 & 0.16 & 0.17 & 0.20 & 0.24 & 0.28 & 0.32 & 0.36 & 0.39 & 0.46 & 0.54 & 0.65 & $\mathbf{0 . 1 0}$ & 0.99 \\
17 & 0.01 & 0.10 & 0.10 & 0.11 & 0.13 & 0.16 & 0.19 & 0.22 & 0.25 & 0.28 & 0.31 & 0.35 & 0.42 & 0.49 & 0.58 & 0.64 & $\mathbf{0 . 1 2}$ \\
\hline
\end{tabular}

The initial laying period of a hen should not be used as a selection criterion, since the heritability estimate for this period is very low (0.04). In P2 (from 23 to 25 weeks of age), a greater estimate $(0.14)$ was observed compared with the other studied periods was obtained; the values for the other studied periods varied from 0.10 to 0.13 . This indicated that P2 (from 23 to 25 weeks of age) could be considered an important period for the selection of laying birds, agreeing with the findings of Wei and van der Werf (1993) and Wolc et al. (2007).

The additive genetic correlation $\left(r_{a}\right)$ and phenotypic estimates $\left(r_{p}\right)$ for the $\mathrm{k}_{\mathrm{a}=3}, \mathrm{k}_{\mathrm{c}=9}$, and $\mathrm{k}_{\mathrm{e}=5}$ model, presented in Table 3, were all positive. The $r_{a}$ varied from 0.10 (between P1 and P17) to 0.99 for all the adjacent periods. The $r_{p}$ varied from 0.01 (between P1 and P17) to 0.65 (between P15 and P16) (Table 3). The P2 genetic correlations with periods from the final third of the cycle were of low magnitude, suggesting that selection made on the basis of this period, where greater heritability was estimated, is not useful for the final cycle periods. Similarly, the other periods had less genetic association among them when they were more distant in time. Such results were also observed for egg production in laying hens by Anang et al. (2000), Szwaczkowski (2003), Luo et al. (2007), and Wolc et al. (2007).

\section{CONCLUSION}

Selection applied in the partial periods of egg production can result in greater genetic 
gains for the adjacent periods. However, as the distance in time between the partial periods of production increases, the selection becomes less efficient. The selection made on the basis of P2, where greater heritability was estimated, is not useful for the final cycle periods.

\section{ACKNOWLEDGMENTS}

The authors are grateful to Embrapa Suínos e Aves and Fundação de Amparo à Pesquisa do Estado de São Paulo (FAPESP). G.C. Venturini, L. El Faro and D.P. Munari received grants from Conselho Nacional de Desenvolvimento Científico e Tecnológico (CNPq); D.A. Grossi and S.B. Ramos received grants from FAPESP. V.A.R. Cruz and C.G. Souza received grants from Coordenação de Aperfeiçoamento de Pessoal de Nível Superior (CAPES) - Programa de Pós-Graduação em Genética e Melhoramento Animal (FCAV/UNESP).

\section{REFERENCES}

Al-Samarai FR, Al-Kassie GA, Al-Nedawi AM and Al-Soudi KAA (2008). Prediction of total egg production from partial or cumulative egg production in a stock of White Leghorn hens in Iraq. Int. J. Poult. Sci. 7: 890-893.

Anang A, Mielenz N and Schüler L (2000). Genetic and phenotypic parameters for monthly egg production on laying hens. J. Anim. Breed. Genet. 117: 407-415.

Anang A, Mielenz N and Schuler L (2001). Monthly model for genetic evaluation of laying hens 1. Fixed regression. $\mathrm{Br}$. Poult. Sci 42: 191-196.

Anang A, Mielenz N and Schuler L (2002). Monthly model for genetic evaluation of laying hens. II. Random regression. Br. Poult. Sci 43: 384-390.

Bignardi AB, El Faro L, Albuquerque LG and Cardoso VL (2008). Modelos de dimensão finita para a estimação de parâmetros genéticos para a produção de leite de primeiras lactações de vacas da raça Holandesa. Ciênc. Rural 38: $1705-1710$

El Faro L, Cardoso VL and Albuquerque LG (2008). Variance component estimates applying random regression models for test-day milk yield in Caracu heifers (Bos taurus Artiodactyla, Bovidae). Genet. Mol. Biol. 31: 665-673.

Fialho FB and Ledur MC (1997). Segmented polynomial model for estimation of egg production curves in laying hens. Br. Poult. Sci. 38: 66-73.

Grossman M, Gossman TN and Koops WJ (2000). A model for persistency of egg production. Poult. Sci. 79: 1715-1724.

Jamrozik J, Schaeffer LR and Dekkers JC (1997). Genetic evaluation of dairy cattle using test day yields and random regression model. J. Dairy Sci. 80: 1217-1226.

Luo PT, Yang RQ and Yang N (2007). Estimation of genetic parameters for cumulative egg numbers in a broiler dam line by using a random regression model. Poult. Sci. 86: 30-36.

Meyer K (1998a). Estimating covariance functions for longitudinal data using random regression model. Genet. Sel. Evol. 30: $221-240$

Meyer K (1998b). DXMRR - A Program to Estimate Covariance Functions for Longitudinal Data by Restricted Maximum Likelihood. In: Proceedings of the 6th World Congress on Genetics Applied to Livestock Production Armidale, Australia, 465-466.

Nuñez-Antón V and Zimmerman DL (2000). Modeling nonstationary longitudinal data. Biometrics 56: 699-705.

Pires AV, Carneiro PLS, Filho RAT, Fonseca R, et al. (2002). Estudo da divergência genética entre seis linhas de aves Legorne utilizando técnicas de análise multivariada. Arq. Bras. Med. Vet. Zootec. 54: 314-319.

Savegnago RP, Caetano SL, Ramos SB, Nascimento GB, et al. (2011). Estimates of genetic parameters, and cluster and principal components analyses of breeding values related to egg production traits in a White Leghorn population. Poult. Sci. 90: 2174-2188.

Szwaczkowski T (2003). Use of Mixed Model Methodology in Poultry Breeding: Estimation of Genetic Parameters, Poultry Genetics, Breeding and Biotechnology. CAB International, Wallingford, 165-202.

Szydlowski M and Szwaczkowski T (2001). Bayesian segregation analysis of production traits in two strains of laying chickens. Poult. Sci. 80: 125-131.

Wei M and van der Werf JH (1993). Animal model estimation of additive and dominance variances in egg production traits of poultry. J. Anim. Sci. 71: 57-65. 
Wolc A, Lisowski M and Szwaczkowski T (2007). Heritability of egg production in laying hens under cumulative, multitrait and repeated measurement animal models. Czech J. Anim. Sci. 52: 254-259.

Wolc A, Barczak E, Wężyk S and Badowski J (2008). Genetic evaluation of production and reproduction traits in two selected lines of geese under multitrait animal model. Anim. Sci. Paper. Rep. 26: 71-78. 